\title{
EPIDEMIOLOGICAL AND PSYCHOSOCIAL ASSESSMENT OF CHILDHOOD OBESITY - PROSPECTIVE STUDY
}

\author{
Oana Elena Iaru, Raluca Maria Vlad, Marina Popov, Roxana Andrei, \\ Irina Dijmarescu, Daniela Pacurar, Dumitru Oraseanu \\ Department of Paediatrics, "Grigore Alexandrescu” Emergency Children's Hospital, \\ Bucharest, Romania
}

\begin{abstract}
Objective. The aim of this study was the evaluation of epidemiological and psychosocial parameters in a sample of overweight and obese children hospitalized for various pathologies. The duration of the study was 12 months. Methods. We performed a prospective study that included overweight and obese children admitted in the Paediatric Unit of our hospital from July 2014 until June 2015. The exclusion criteria consisted of the presence of secondary pathologies related to obesity. A questionnaire was applied to all patients included with questions about family and personal risk factors for obesity; we realised also a psychological evaluation and quality of life assessment (PedsQL). For each patient we performed clinical evaluation, laboratory and imaging investigations (abdominal ultrasound).

Results. The study included 78 patients: 27 overweight (34.6\%), 51 obese (65.3\%). We studied the family history concerning the weight status: 55 had one obese parent and 15 had both parents with obesity. We identified dietary errors for almost all patients $(65$ patients $=83.3 \%$ ). The average number of hours of physical activity was rather low ( 0.57 hours/day) and the average time spent in front of the TV and computer was increased (5.7 hours/day).

Conclusions. Obesity has been associated with familial background of obesity, dietary errors (both eating schedule and composition), decreased time spent doing physical activity and increased time dedicated to computer and television. These conclusions should be considered in the prophylactic and curative programs done for this pathology.
\end{abstract}

Keywords: obesity, pediatric population, dietary errors, physical activity

\section{INTRODUCTION}

Obesity is a global issue of the adult population, both in developing countries and especially in developed ones. According to World Health Organization statistics, obesity prevalence has almost doubled in the period 1980-2008 (1), approximately $50 \%$ of the adult population of Europe is defined as overweight (according to weight charts). This pattern is more and more applicable to the pediatric population: American data show that in the last 30 years the prevalence of obesity has doubled among children, the statistics for 2012 revealing that more than a third of children are overweight/obese (2); the same context is true for Europe, where one of three children under 11 years is overweight or obese (3).

Urbanization, unhealthy diet and physical inactivity are major factors increasing child obesity, especially in developed countries (4). Predisposing factors for obesity are found in the family (the majority of obese children having a family history of obesity) and society (fast-food networks as part of weakly eating schedule, increased amount of time spent in front of computer or TV). The result is often a child with health problems in the present and especially in the future, with low self-esteem, without friends and not integrated into community.

Corresponding author:

Oana Elena laru, Institute of Oncology, 252 Fundeni Street, Bucharest

E-mail: oana_iaru@yahoo.com 


\section{MATERIAL AND METHOD}

We performed an observational, prospective study; we included children aged between 6 and 18 years, who were overweight (BMI greater than 85 th percentile) or obese (BMI greater than the 95th percentile) admitted to the pediatric service of SCUC "Grigore Alexandrescu" Bucharest during July 2014 - June 2015.

Patients were selected after admission for other pathology than obesity (usually an acute illness). Selection criteria were the anthropometric parameters (primarily BMI greater than the $85^{\text {th }}$ percentile for gender and age).

We applied a questionnaire for each patient; the items of the questionnaire were about: family history (heart disease, metabolic syndrome, liver disease, type II diabetes, obesity), nutritional status in the family (especially nutritional status of parents), analysis of food intake and composition of the meals, assessment of physical activity made at home and at school, assessment of time spent in front of TV or computer, psychological evaluation (conflicts in the family, conflicts at school), a history of pathological antecedents (respiratory, migraine). The second activity was to evaluate: anthropometric indices, physical examination, specific laboratory investigations (renal and liver function, assessment of lipid, protein and carbohydrate metabolism). An abdominal ultrasound (to identify steatosis) was performed for each patient. Psychological evaluation was realized using questionnaires to assess quality of life (PedsQL) (5).

Data were recorded and statistically processed with Microsoft Excel.

The study was approved by the Ethics Committee of SCUC "Grigore Alexandrescu", for each enrolled patient the parents signed a consent form.

\section{RESULTS}

The study group was formed of a total of 78 patients aged 6 to 18 years, 33 girls and 45 boys, mean age 138 months (11 years and 6 months). Depending on body mass index, 51 patients $(65.38 \%)$ are obese (BMI greater than the 95th percentile for gender and age) and 27 patients (34.62\%) are overweight (BMI between percentiles 85 and 95 suitable for age and sex).

Many patients had positive family nutritional status for overweight/obesity - 58 children, respectively $74.35 \%$ (targeted questions were addressed to select only close relatives with whom the child has frequent contact/spends a large amount of time respectively family members who could be role models). Regarding the nutritional status of the parents, the results are as follows: 55 children have an overweight/obese parent and 15 of them have both parents with overweight/obesity (Fig. 1).

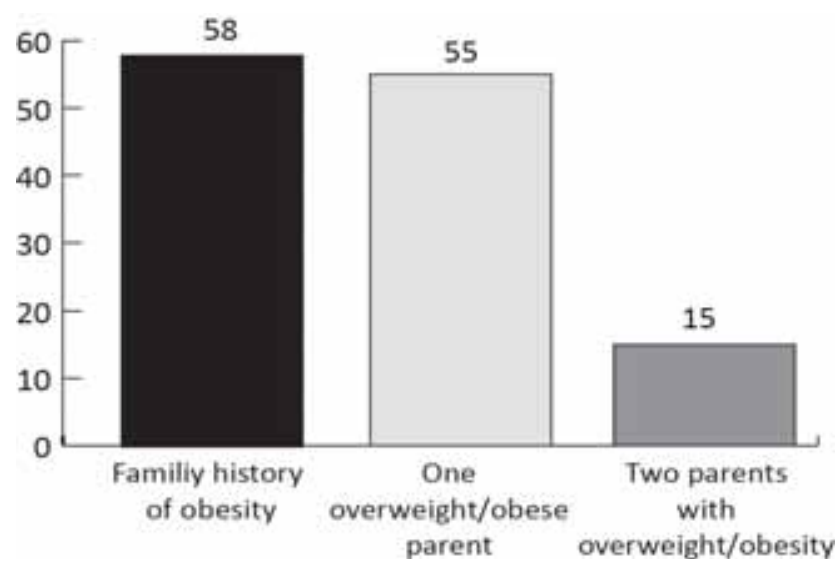

FIGURE 1. Family history of obesity

Analyzing data regarding the pattern of alimentation from the questionnaire we applied showed dietary errors for the vast majority of patients (65 of the 78 patients, $83.3 \%$ respectively). Errors arise both in eating patterns (chaotic meals, disorganized program $-42.3 \%$; absence of main meals, meals at inappropriate hours $-28.2 \%$, frequent snacks $61.5 \%$ ) but also in terms of composition of meals: excessive carbohydrates (from sweets, but also from bread, pasta) are part of the diet of 61 children in the study group $(78.2 \%)$ and fried foods and saturated fats are found excessively in the daily diet of 32 children (41\%). Soft drinks are consumed daily by 43 children $(55.1 \%)$. Regarding fast food most children receive occasionally, but 29 of them (37.1\% respectively) eat fast food on a regular basis (at least once a week) - data represented in Fig. 2.

Another chapter in the style of life analysis of the overweight/obese patients is the physical inactivity and the amount of time spent watching TV, in front of computer and other devices (tablet, smart phone) plus the amount of time devoted to computer games. Average time spent in front of computer or with computer games is 3.2 hours/day or 22.4 hours/week (Fig. 4 and Fig. 5), and the average time spent watching TV is 2.5 hours/day, respectively 17.5 hours/week (Fig. 3).

In contrast with sedentary activities, physical activity is not an important part of daily/weekly schedule for children. Only 42 children practice a form of daily physical activity (sports in an organized form, recreational activities or even just play involving movement). The average number of hours of sport per week practiced by children in- 


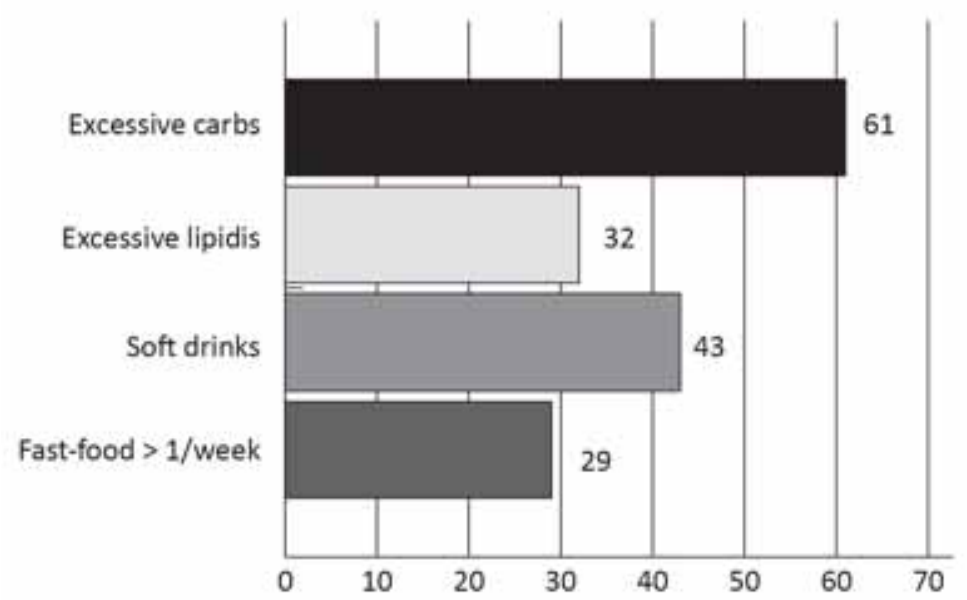

FIGURE 2. Dietary errors

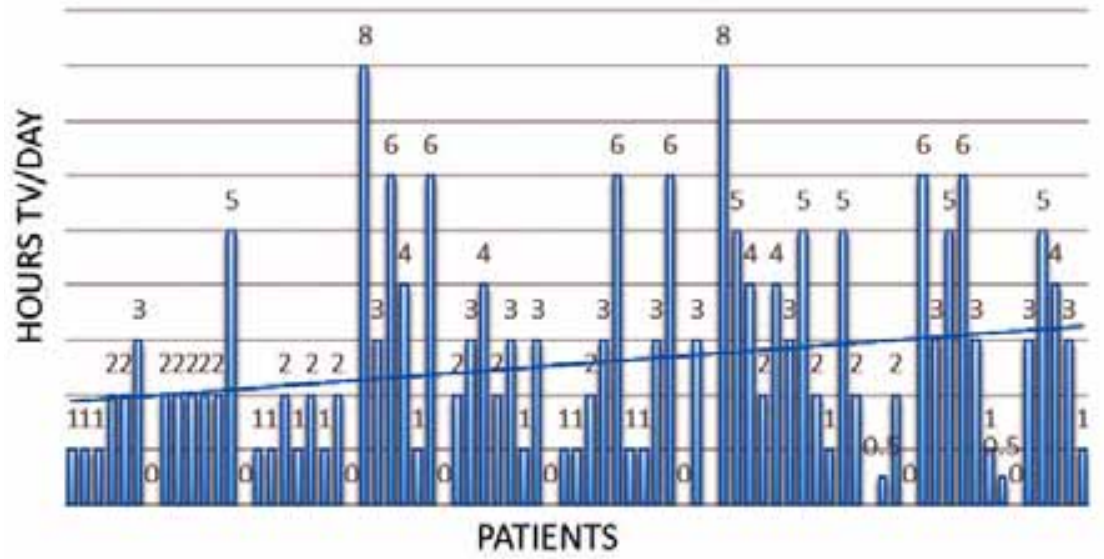

FIGURE 3. Watching TV (hours/day) - average 2.5 hours/day

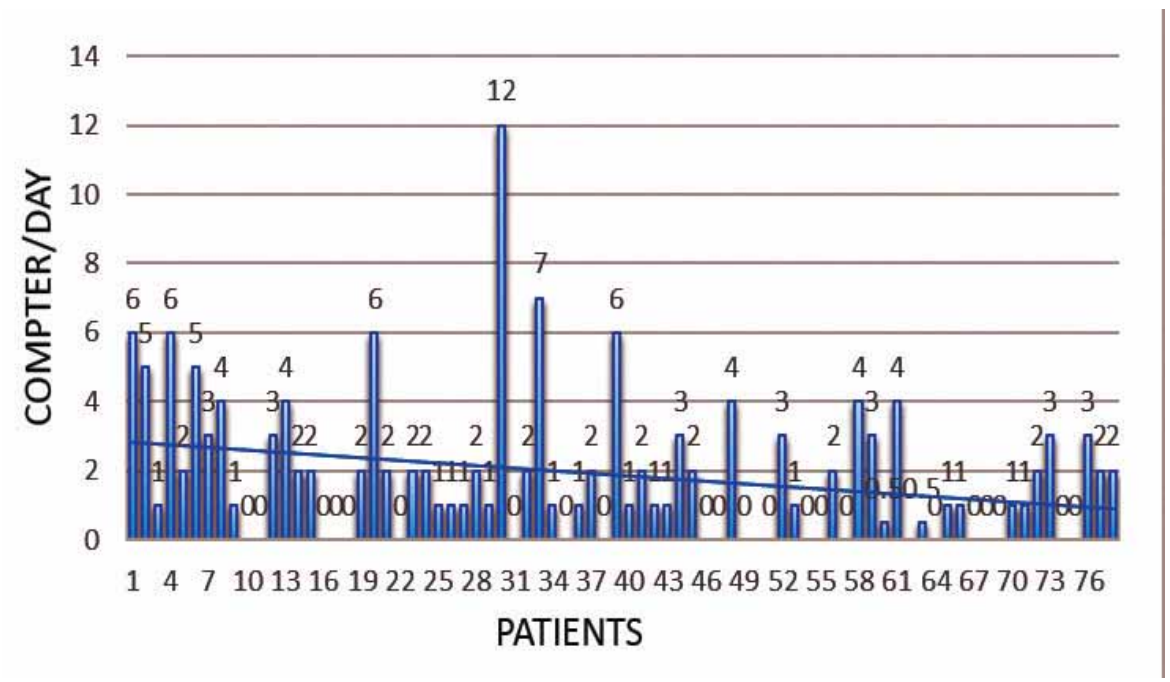

FIGURE 4. Hours spent in front of computer/day (average 1.8 hours/day)

cluded in the study is 2.9 ( 0.4 hours/day). In addition we have the physical education classes at school (average1.2 hours/week). It's important to observe that $23(29.4 \%)$ of the patients in the study did not practice physical education at school.

Although the family may not be aware of the frequent conflictual relations or social problems for the children included in the study, by applying special questionnaires we found that 30 patients $(38.46 \%)$ had conflictual relations with one or more of the family members (parents, siblings). Regarding relations with children of same age and schoolmates, 9 children (11.5\%) said they fail to make friends and $13(16.6 \%)$ don't feel integrated at school. 


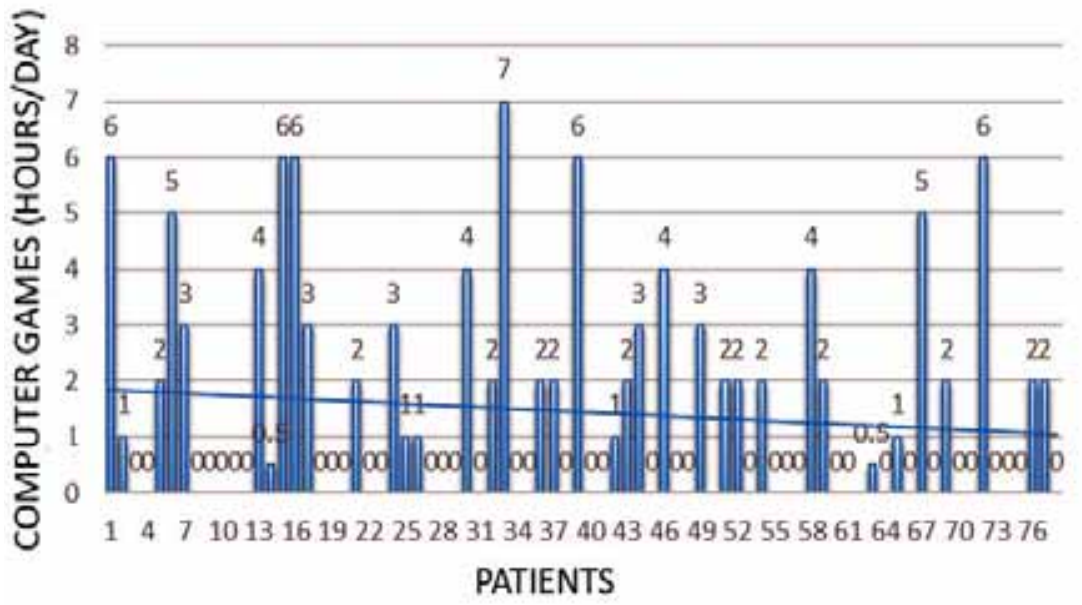

FIGURE 5. Hours spent with computer games/day (average 1.4 hours/day)

\section{DISCUSSIONS}

Obesity, a public health problem relatively recent in the Romanian society, is more and more actual for the pediatric population (according to a study from 2014 within the European Childhood Obesity Surveillance Initiative - COSI, a quarter of Romanian children of 8 years old are overweight or obese) (6). Although there are no national data for all age groups, the prevalence of this pathology among children hospitalized for other acute illnesses is increasing.

Obesity in the family, among relatives that could become role models to children and especially among parents, has an important role in the etiology of child obesity. According to the literature a child with an obese parent has a $50 \%$ chance of becoming obese, and if both parents are obese, the chances increase to $80 \%$ (4). Another study (7) shows that parental obesity increases more than half the chances of a child to become obese. In our study 55 children $(70.5 \%)$ have a parent with overweight/obesity and 15 children $(19.23 \%)$ have both parents with overweight/obesity.

Obesity is the imbalance between the amount of calories gained compared to the amount of calories spent (8). The amount of useless calories ("empty calories") which will later participate in the formation of extra-weight represents about $40 \%$ in the diet of children aged between 2-18 years. The sources of these calories are: soft drinks, desserts/ snacks, pizza, whole milk (9). In our population 61 children $(78.2 \%)$ consume excessive carbohydrates and 32 children (41\%) have high amounts of saturated fats in the daily diet. Regarding soft drinks they are part of the daily diet for 43 children (55\%).

Eating fast food at least once a week is associated with increased risk of obesity (10). In our study group 29 children $(37.1 \%)$ eat fast food weekly.
In an evaluation of an American population of adolescents for 2013 only $29 \%$ of the participants practiced physical activity of any kind for at least 60 minutes a day, 7 consecutive days anterior to the study (recommendations of the World Health Organization regarding physical activity for children are: 60 minutes of moderate physical activity daily) $(11,12)$. For our population the average daily physical activity is reduced to 0.4 hours. It's important that only 42 children in the study are doing a form of physical activity daily.

Regarding physical inactivity (time spent in front of TV and computer), a study done in 2011 on an American population of 12,650 children in kindergarten showed that spending more than an hour in front of TV or computer each day leads to a risk of becoming overweight increased by $52 \%$; other conclusion of this study was that the average time children spend in front of TV/computer daily is 3.3 hours (13). In the population we studied the average daily time spent in front of TV is 2.5 hours and in front of computer is 3.2 hours - significantly longer than the time allocated to physical activity.

\section{CONCLUSIONS}

In the pediatric population we studied obesity is associated with a family history of this condition, especially among parents. Dietary investigation revealed errors in most patients, both in terms of patterns of meals and quantity of food, but especially regarding the food composition. Eating fast-food is a regular habit for most participants in the study, one third of them consuming these products more than once a week. Average time of physical activity is very low; in contrast, the time dedicated to computer/TV is rather increased. 


\section{REFERENCES}

1. WHO official data: http://www.cdc.gov/healthyschools/obesity/facts. $\mathrm{htm}$

2. WHO official data: http://www.euro.who.int/en/health-topics/ noncommunicable-diseases/obesity/data-and-statistics

3. www.aacap.org (American Academy of Child and Adolescent Psychiatry)

4. www.pedsql.org

5. Health Ministry press release from 14.07.2014, part of European Childhood Obesity Surveillance Initiatiev - COSI

6. Whitaker R., Wright J., Pepe M., Seidel K., Dietz W. Predicting Obesity in Young Adulthood from Childhood and Parental Obesity, N. Eng. J. Med, 1997, 337, 869-73

7. Daniels S.R., Arnett D.K., Eckel R.H., et al. Overweight in children and adolescents: pathophysiology, consequences, prevention, and treatment, Circulation 2005; 111; 1999-2002.
8. Reedy J., Krebs-Smith S.M. Dietary sources of energy, solid fats, and added sugars among children and adolescents in the United States, Journal of the American Dietetic Association 2010; 110:1477-1484.

9. Dietary Guidelines Advisory Committee. Report of the Dietary Guidelines Advisory Committee on the Dietary Guidelines for Americans, 2010, to the Secretary of Agriculture and the Secretary of Health and Human Services. Washington, DC: U.S. Department of Agriculture; 2010

10. WHO - Global recommendations on physical activity for health

11. CDC. Youth Risk Behavior Surveillance - United States, 2013. MMWR 2014; 63(SS-4)

12. The Early Childhood Longitudinal Study, Kindergarten Class of 2010-11 (ECLS-K:2011) 